\title{
Microscopia confocal reflectante aplicada ao diagnóstico do melanoma cutâneo
}

\author{
Reflectance confocal microscopy in the diagnosis of cutaneous melanoma
}

\author{
Cintia Rito ${ }^{1}$
}

\author{
Juan Pineiro-Maceira ${ }^{2}$
}

\begin{abstract}
Resumo: O melanoma cutâneo é um problema de saúde pública a nível mundial. Sua incidência tem aumentado, de forma marcante, nos últimos anos, e o diagnóstico e excisão precoces são essenciais para o bom prognóstico dos pacientes. Neste contexto, a dermatoscopia ganhou grande importância, nas últimas duas décadas, melhorando, de forma significativa, a acurácia do diagnóstico do melanoma, em estágios iniciais. Porém, existem algumas lesões benignas que apresentam dermatoscopia duvidosa, levando à realização de cirurgias desnecessárias. Mais recentemente, a microscopia confocal reflectante vem sendo introduzida como método diagnóstico auxiliar promissor, por ser um exame não-invasivo, realizado in vivo, de forma simples, indolor e de rápida execução. É a única técnica capaz de identificar estruturas celulares e examinar a epiderme e a derme papilar, com resolução semelhante à da histopatologia, com uma sensibilidade de $97,3 \%$, e especificidade de $72,3 \%$ para o diagnóstico do melanoma cutâneo. É uma importante ferramenta diagnóstica, visto que não substitui o exame histopatológico realizado no pós-operatório, mas permite a abordagem racional das lesões com dermatoscopia duvidosa, evitando procedimentos cirúrgicos desnecessários.
\end{abstract}

Palavras-chave: Diagnóstico; Melanoma; Microscopia confocal

\begin{abstract}
Skin melanoma is an international public health issue, with a considerable increase in frequency over the past few years. Early diagnosis and excision are essential for good patient prognosis. Over the past two decades dermoscopy has gained significance due to a major improvement in the accuracy of skin melanoma diagnosis in its early stage. However, there are some benign lesions of questionable dermoscopy, which may lead to the performance of unnecessary surgery. Recently, reflectance confocal microscopy has been introduced as a promising supplementary diagnostic method. It is a noninvasive, in vivo, simple, painless and quick exam. It is the only technique capable of identifying cellular structures and to examine the epidermis and papillary dermis with a resolution similar to that of histopathology, with a sensitivity of $97.3 \%$ and specificity of $72.3 \%$ in the diagnosis of cutaneous melanoma. This is an important diagnostic tool, because it does not substitute post-surgical histopathological examination and allows for the rational assessment of lesions of questionable dermoscopy, thus avoiding unnecessary surgical procedures.

Keywords: Diagnosis; Melanoma; Microscopy,confocal
\end{abstract}

\section{INTRODUÇÃO}

O melanoma e o câncer de pele não-melanoma são, hoje, os mais comuns tipos de câncer na população de cor branca, segundo alguns autores. ${ }^{1}$ Ambos os tumores têm demonstrado um aumento de incidência, em todo o mundo. O melanoma cutâneo é o câncer o qual cresce mais rapidamente na população caucasiana, e sua morbidade tem aumentado dramaticamente, nos últimos anos. ${ }^{1-5}$
Existe uma forte correlação inversa entre: a taxa de sobrevida e a espessura do tumor, já que não existem terapias efetivas, curativas para o melanoma avançado. O diagnóstico precoce e a excisão são essenciais para reduzir a morbidade e aumentar a sobrevida do paciente. ${ }^{5-8}$ Há relatos de que este aumento de incidência se deva, principalmente, à crescente detecção de melanomas iniciais (com espessura menor). O

Aprovado pelo Conselho Editorial e aceito para publicação em 21.09.09.

* Trabalho realizado na Universidade Federal do Rio de Janeiro (UFRJ) - Rio de Janeiro (RJ), Brasil.

Conflito de interesse: Nenhum. / Conflict of interest: None

Suporte financeiro: Nenhum. / Financial Funding: None

Mestranda pela Universidade Federal do Rio de Janeiro (colaboração da Universidade degli studi di Reggio-Emilia - Modena)

Preceptora de Dermatoscopia do curso de residência Médica do Hospital Geral de Bonsucesso - Rio de Janeiro (RJ), Brasil.

Pós-Doutorado pelo Armed Forces Institute of Pathology, Estados Unidos.

Professor Adjunto da Universidade Federal do Rio de Janeiro - Rio de Janeiro (RJ), Brasil. 
aperfeiçoamento dos métodos diagnósticos torna frequente a identificação de pacientes de baixo risco (isto é, com espessura de Breslow menor que $0,76 \mathrm{~mm}$ ) e esta é a principal razão para que não haja alteração na taxa de mortalidade relacionada a esta neoplasia. 2,9

A preocupação em realizar o diagnóstico precoce dessas lesões estimula o desenvolvimento constante de diferentes técnicas diagnósticas não-invasivas. ${ }^{10,11}$

O desenvolvimento da dermatoscopia, como método auxiliar diagnóstico, possibilitou o aumento da acurácia diagnóstica do melanoma, em até 90\%,12,13 com uma maior sensibilidade e especificidade, em comparação ao diagnóstico clínico isolado. ${ }^{5,14} \mathrm{E}$ hoje, contamos também com o auxílio da microscopia confocal, modo reflectante. ${ }^{15}$

De todos os novos métodos na diagnose nãoinvasiva, a microscopia confocal, modo reflectante, é a única técnica que permite o exame da epiderme e derme papilar, com resolução próxima a do exame histopatológico, identificando estruturas microanatômicas e células individuais com alta resolução. 16,17 Imagens confocais têm sido avaliadas como qualitativamente e quantitativamente, correspondentes a cortes histológicos horizontais. 11,18-24

A microscopia confocal, modo reflectante, e a dermatoscopia obtêm imagens no plano horizontal. Isto facilita a correlação entre as imagens obtidas nos dois exames, já tendo sido descrita a correlação entre alguns padrões globais e locais dermatoscópicos e suas características microscópicas confocais. ${ }^{25-28}$

\section{HISTÓRICO}

O microscópio confocal foi inventado por Marvin Minsky em 1957.10,29,30 Entretanto, foi necessário o desenvolvimento de uma fonte de luz adequada e tecnologia computadorizada, para que fosse possível a sua utilização na pele humana, in vivo. $O$ primeiro relato do uso do microscópio confocal modo reflectante in vivo, na pele humana, ocorreu em 1995. 31

\section{Avaliação Diagnóstica com a Microscopia Confocal Reflectante}

Esta nova metodologia diagnóstica apresenta inúmeras vantagens - como permitir a identificação instantânea das estruturas da pele, por ser um procedimento não-invasivo, realizado in-vivo, indolor, não causando dano tecidual. A pele não é alterada por processamento do material, minimizando a ocorrência de artefatos; os dados são coletados em tempo real, permitindo a análise rápida da lesão em questão, e o exame pode ser repetido no sítio da pele, em questão inúmeras vezes. Dessa forma, podem-se avaliar alterações que ocorram na lesão, de forma dinâmica, como em resposta à alguma terapia utilizada, e as bordas da lesão podem ser avaliadas in vivo, permitindo um melhor planejamento cirúrgico.32-34 Com o incremento da teledermatologia, este exame poderá ser analisado por diversos especialistas, com relativa facilidade, e o tempo necessário para a sua realização não é superior ao necessário para a realização da dermatoscopia digital. 10,11,31,16

Contudo, uma das limitações da microscopia confocal, modo reflectante atual, é a possibilidade de analisarmos as estruturas microanatômicas, somente até a profundidade de $350 \mu \mathrm{m}$, o que significa, geralmente, $o$ alcance apenas até a derme papilar. Por isso, alterações na derme reticular e os tumores que invadem a profundidade não podem ser avaliados adequadamente. ${ }^{16}$ Entretanto, em áreas onde a epiderme apresenta pouca espessura, parte da derme reticular, pode também ser avaliada.

Diversos tipos de aparelhos já foram fabricados e dois modelos foram comercializados recentemente: o vivascope 1500 (Figura 1), em 2000, e o vivascope 3000 , em 2006. Este possui uma pistola que facilita a realização do exame.

O vivascope utiliza o laser de diodo, com comprimento de onda de $830 \mathrm{~nm}$ e lentes objetivas de $30 \mathrm{x}$, com abertura numérica de $0,9 \mathrm{com}$. O que conseguimos uma resolução lateral de 1-2 $\mu \mathrm{m}$ e resolução axial de $3-5 \mu \mathrm{m}$. A penetração da profundidade da imagem varia de 200-500 $\mu \mathrm{m}$ (em média $350 \mu \mathrm{m}$ ), permitindo a visualização da epiderme e derme superior. A potência do laser é menor que $30 \mathrm{~mW}$ e não causa dano à pele ou aos olhos. 11,31,16

Este aparelho emite uma luz que ilumina um pequeno ponto, no interior do tecido. Esta é refletida, e passa através de uma pequena abertura, chamada

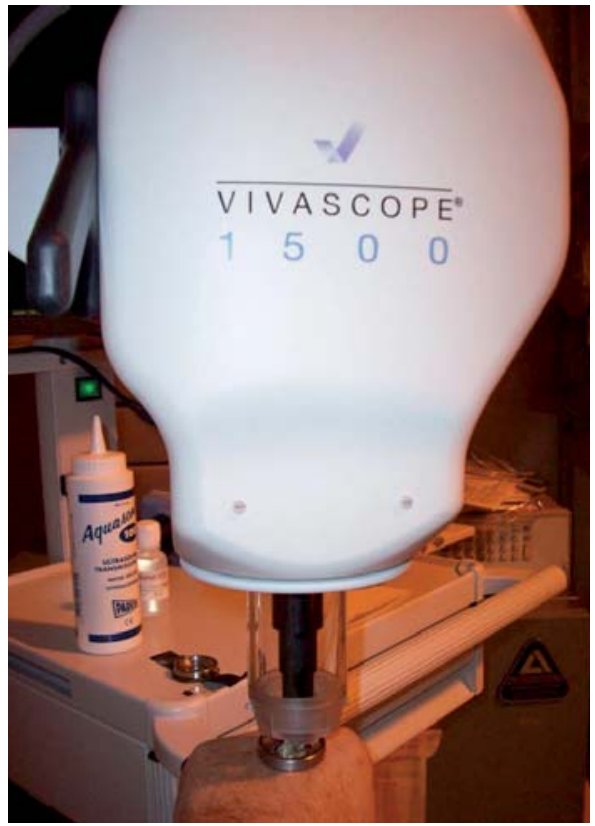

Figura 1:

Vivascope 1500 
"pinhole" e, a seguir, a imagem é formada no detector. Esta abertura não permite que a luz refletida (reflectante), a partir de outro ponto do tecido, alcance o detector. Desta forma, somente a luz refletida, a partir da região que está no foco (confocal), é detectada. Para se criar a imagem da lesão inteira, cada ponto é "escaneado". 31

O instrumento utiliza lentes objetivas, de imersão em água, sendo o índice de refração da água e o da epiderme. ${ }^{1,34}$ Isto minimiza aberrações esféricas, porque também é utilizado gel à base de água, como meio de imersão, particularmente, se a lesão for descamativa ou hiperceratótica, pois o gel reduz as irregularidades da refração.

O exame é realizado de forma simples, indolor e rápida. Utilizamos um anel de metal, sendo fixado na pele do paciente, com o auxílio de um adesivo. No interior deste anel, adicionamos água ou gel, logo ocorre a fixação deste anel ao aparelho, por meio magnético.

Existem três diferenças básicas da microscopia confocal, em relação à rotina histológica convencional: na microscopia confocal, as imagens são obtidas no sentido horizontal da lesão, enquanto na histologia convencional, os cortes são realizados no sentido vertical. Essas imagens são obtidas em uma escala de cinza, semelhante às que ocorrem nas radiografias. 31 Além disto, podemos obter imagens estáticas e dinâmicas da pele, estas podem ser gravadas em um videotape de 20-30 Hz, demonstrando eventos como o fluxo sanguíneo. ${ }^{31}$

As imagens são formadas pelo microscópio confocal através de diferentes tons de brilho. O mecanismo de contraste de brilho, na microscopia confocal, é causado por diferenças na refração da luz. Na escala de cinza da microscopia confocal, as estruturas surgidas com maior brilho possuem um alto índice de refração, comparadas com as estruturas ao seu redor. Componentes da pele, com alto índice de refração são: melanina $(n=1,72)$, queratina $(n=1,51)$ e colágeno $(n=1,43)$. Esses componentes aparecem brilhantes, circundados pela epiderme $(n=1,34)$ e derme $(\mathrm{n}=1,41) .10$

\section{Exame da pele normal}

Para interpretarmos as imagens da pele anormal, é necessário familiarizar-se com a aparência da pele normal. Na histologia da pele normal, a epiderme é composta primariamente de: ceratinócitos e uma menor população de células dendríticas; sendo os melanócitos e as células de Langerhans. A derme é composta por: vasos sanguíneos, nervos, células inflamatórias e fibroblastos envolvidos por fibras de colágeno e elastina. A derme papilar forma projeções para $\mathrm{o}$ interior da epiderme, chamadas papilas dérmicas. $\mathrm{O}$ ponto de encontro entre a epiderme e a derme é chamado de: junção dermo-epidérmica. Na epiderme, os ceratinócitos se diferenciam para constituírem quatro camadas distintas. A microscopia confocal, modo reflectante, permite a identificação de cada uma destas camadas baseada em características arquiteturais e citológicas, bem como, na medição da profundidade do corte, à qual se relaciona a localização de cada camada. ${ }^{10,31}$

A camada córnea é a mais superficial da epiderme, na qual se encontra a $0-15 \mu \mathrm{m}$ de profundidade, e é composta por ceratinócitos achatados, anucleados. ${ }^{10}$ Esta camada varia de espessura, dependendo do sítio anatômico e da exposição solar. À microscopia confocal, ela produz uma imagem brilhante. ${ }^{31,17}$ Grandes corneócitos $(25-50 \mu \mathrm{m})$, poligonais, anucleados são vistos. Os dermatoglifos aparecem como vales lineares escuros entre grupos de corneócitos (Figura 2). 10,31

A camada granulosa encontra-se a $10-20 \mu \mathrm{m}$ de

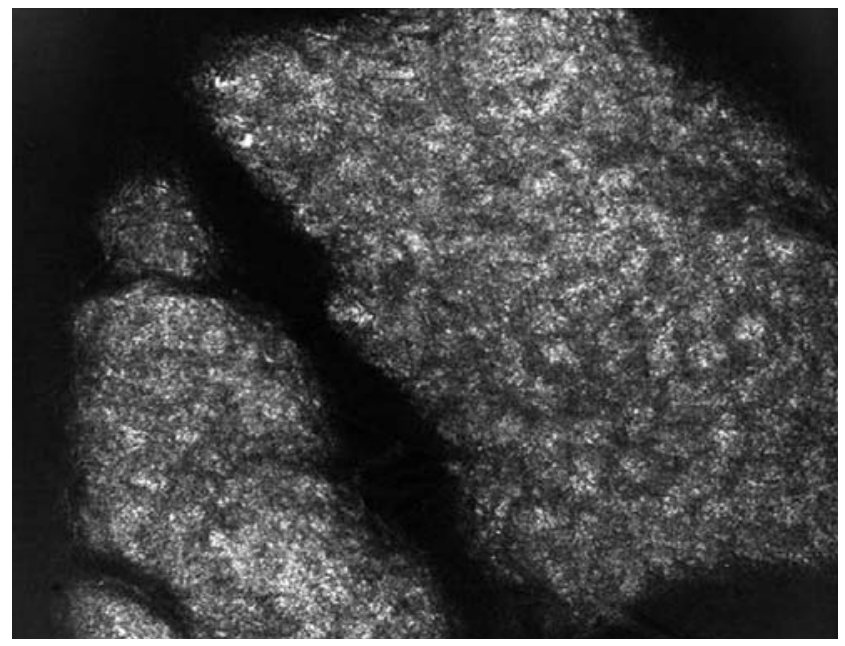

Figura 2: Camada Córnea compostas por corneócitos poligonais, anucleados

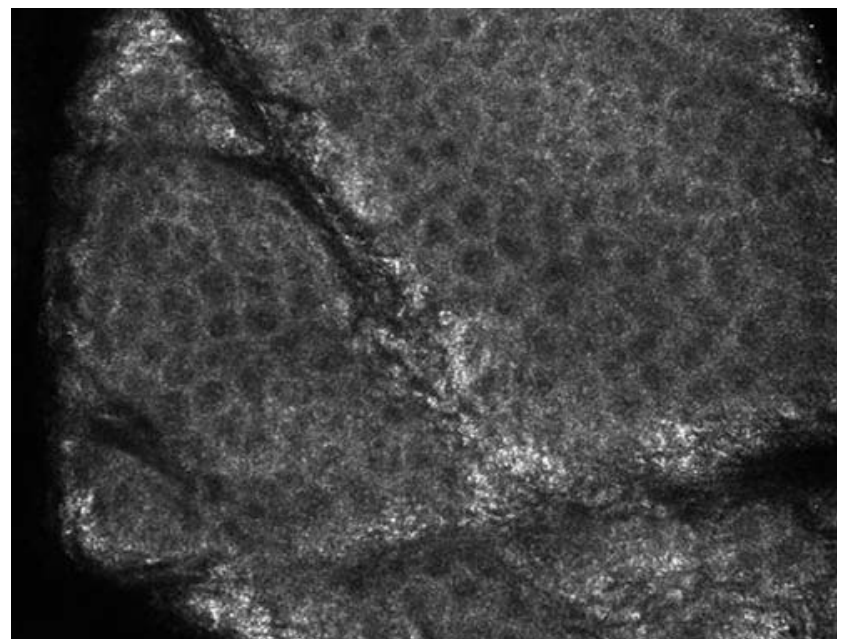

Figura 3: Camada Granulosa composta por ceratinócitos dispostos no padrão em favo de mel 
profundidade e é composta por ceratinócitos poligonais, contendo grânulos de cerato-hialina, no citoplasma e núcleo grande e oval. À microscopia confocal, este ceratinócito mede de $25-35 \mu \mathrm{m}$ e se apresenta como uma célula achatada, com área oval central escura, correspondendo ao núcleo, circundada por citoplasma brilhante e granuloso. Comumente no interior do núcleo escuro, é visto um pequeno ponto central branco: o nucléolo. O citoplasma aparece brilhante, por conta das numerosas estruturas, com índice de refração de $0,1-1 \mu \mathrm{m}$, correspondendo as organelas e aos grânulos de cerato-hialina. Os ceratinócitos possuem as bordas bem demarcadas e estão dispostos em padrão de favo de mel (Figura 3). ${ }^{10,11,31}$

A camada espinhosa encontra-se a aproximadamente $20-100 \mu \mathrm{m}$ de profundidade, a partir do estrato córneo, no qual apresenta aproximadamente 5 a 10 ceratinócitos em sua espessura, com formato poligonal e, progressivamente, achatam-se em direção à superfície. Ao exame, estes possuem aproximadamente 15-25 $\mu \mathrm{m}$ de dimensão, com forma poligonal, contendo uma área central escura oval ou redonda, correspondendo ao núcleo, e um citoplasma de contorno brilhante. Estes ceratinócitos apresentam a borda claramente demarcada e estão dispostos em padrão de favo de mel, como na camada anterior (Figura 4). ${ }^{10,31}$

A camada basal é encontrada a aproximadamente $40-130 \mu \mathrm{m}$ de profundidade, a partir da superfície. É uma camada composta de somente uma fileira de ceratinócitos colunares, na qual contém melanócitos distribuídos de forma regular; em proporção de aproximadamente 1 melanócito para 10 ceratinócitos. A melanina é a maior fonte de contraste na epiderme para a microscopia confocal modo reflectante. Os ceratinócitos aparecem como células brilhantes, medindo em torno de 7-12 $\mu \mathrm{m}$. Quando obtemos uma

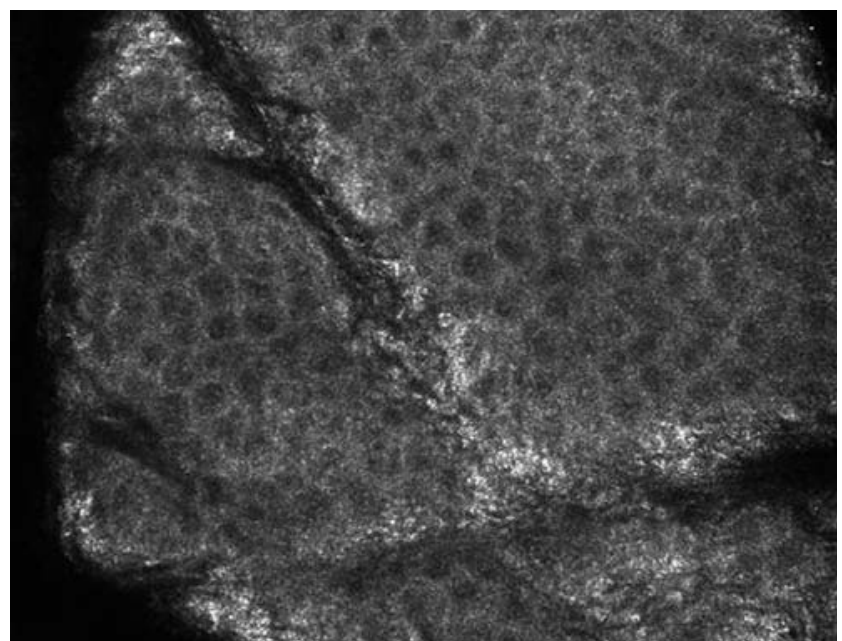

Figura 4: Camada Granulosa composta por ceratinócitos dispostos no padrão em favo de mel imagem um pouco mais profunda: a porção suprapapilar, da junção dermoepidérmica, aparece como anéis de células basais, circundando a papila dérmica escura, a qual frequentemente demonstra fluxo sanguíneo e colágeno. ${ }^{10,31}$ Esse padrão papilar oval é característico das lesões benignas (Figura 5).

A detecção dos melanócitos normais só é possível raramente, já quando são identificados, apresentam-se como estruturas ovais ou redondas, na junção dermoepidérmica e com ramificações externas, podendo corresponder aos seus dendritos. ${ }^{31}$ Do quarto dia ao oitavo dia, após a exposição solar, os melanócitos dendríticos podem ser melhores observados, isto ocorre porque o brilho nas capas de melanina, na camada basal, diminui. A partir do vigésimo nono dia, o intenso brilho das capas de melanina reaparece, impedindo, novamente, a visualização destes melanócitos. ${ }^{11,35}$

A junção dermoepidérmica é composta pelas cristas epidérmicas e papilas dérmicas, e corresponde ao ponto de união entre a epiderme e a derme. A derme papilar encontra-se a 50-150 $\mu \mathrm{m}$ de profundidade, e inclui a papila dérmica e uma pequena camada, abaixo da papila dérmica, indo até o plexo vascular superficial. Ela aparece escura, com fibras colágenas cinzas, e é circundada por um anel de ceratinócitos brilhantes. As fibras colágenas podem aparecer como uma malha reticulada de fibras, medindo 1-5 $\mu \mathrm{m}$ de diâmetro (Figura 6), ou como bandas brilhantes medindo 5-25 um de diâmetro (essas bandas são mais características da derme reticular). Com o artifício da captura por vídeo, podemos ver o fluxo sanguíneo através dos capilares. 10

A derme reticular situa-se entre: o plexo sanguíneo superficial e o subcutâneo, a uma profundidade maior que $150 \mu \mathrm{m}$. À microscópia confocal, modo

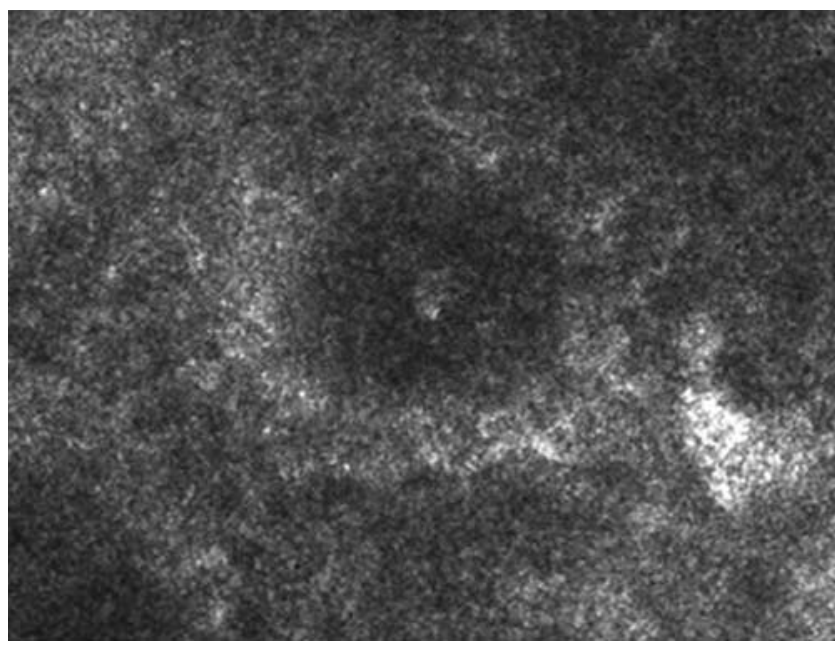

Figura 5: Junção dermo-epidérmica 


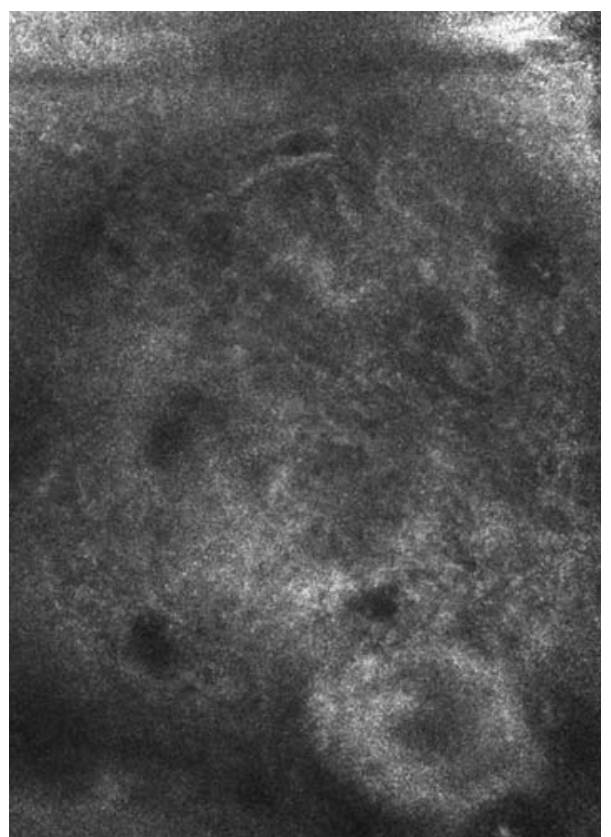

Figura 6:

Derme papilar, apresentando

fibras

colágenas finasa

reflectante, é de difícil visualização, com exceção da sua porção superior, sendo vista em algumas situações específicas. Quando vista, ela revela bandas espessas de colágeno, de coloração cinza, arranjadas em um padrão fascicular, entremeado por lumens escuros, correspondendo a vasos sanguíneos, e poucas células brilhantes, correspondendo a células inflamatórias. ${ }^{10}$

\section{Características microscópicas confocais relacionadas ao melanoma}

No diagnóstico do melanoma cutâneo, pode-se alcançar uma sensibilidade de $97,3 \%$ e uma especificidade de $72,3 \% .36$ Os critérios utilizados para realizar este diagnóstico são: $15,36-40$

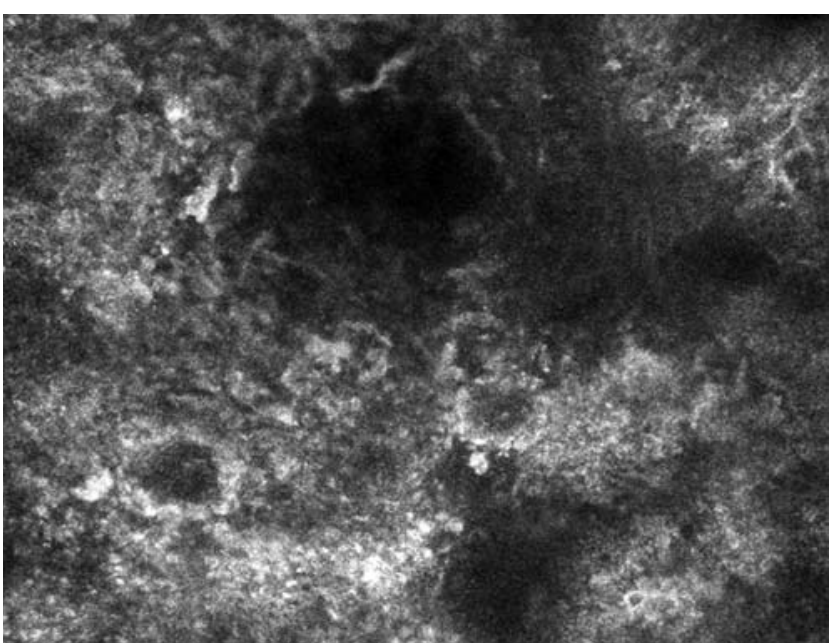

Figura 7: Melanócitos atípicos ao nível da camada basal (células redondas brilhantes com núcleo escuro) e perda do contorno papilar normal

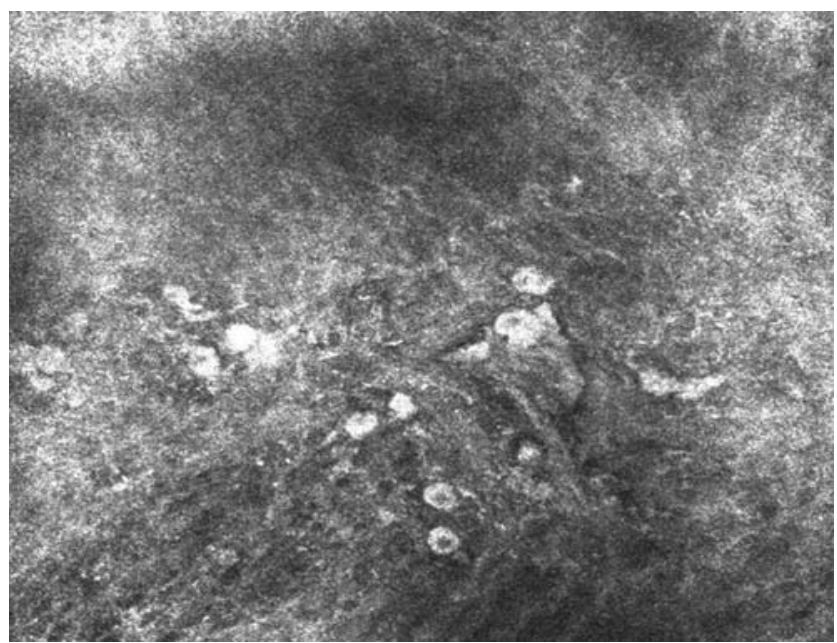

Figura 8: Infiltração pagetóide- presença de melanócitos atípicos (células redondas brilhantes com núcleo escuro) nas camadas superiores

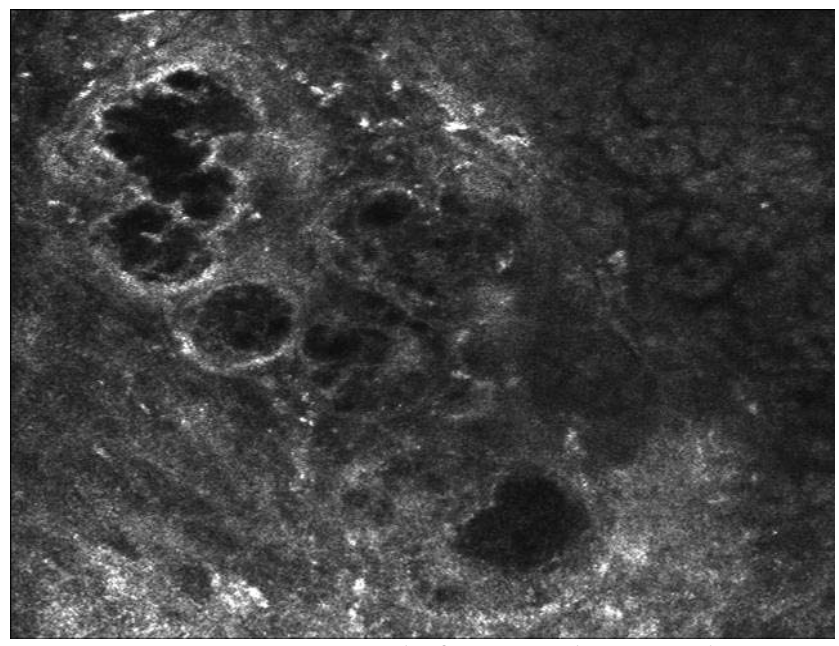

FigURA 9: Estrutura cerebriforme em derme papilar

- Atipia citológica importante na camada basal (Figura 7);

- Perda do contorno oval das papilas na junção dermo-epidérmica (Figura 7);

- presença de células arredondadas, brilhantes nas camadas superficiais (Figura 8);

- células melanocíticas em franca ascenção pagetóide por toda a lesão. São células pleomórficas, nucleadas com citoplasma brilhante;

- grupamentos celulares confluentes, com células pouco brilhantes, configurando um aspecto cerebriforme característico, no interior da derme papilar (Figura 9);

- presença de células isoladas, nucleadas, no interior da derme papilar, correspondendo à melanócitos (Figura 10). 


\section{CONCLUSÃo}

A microscopia confocal é uma metodologia promissora, através da qual poderemos compreender inúmeros aspectos patológicos e fisiológicos cutâneos. É capaz de examinar a epiderme e derme, avaliando suas estruturas teciduais em tempo-real, monitorando processos dinâmicos. Suas aplicações incluem a pesquisa clínica, bem como o diagnóstico de diversas condições, inclusive o melanoma cutâneo com sensibilidade de $97,3 \%$ e especificidade de $72,3 \%$ nesta doença. Sendo, desta forma, uma importante ferramenta diagnóstica, auxiliar à dermatoscopia e à histopatologia.

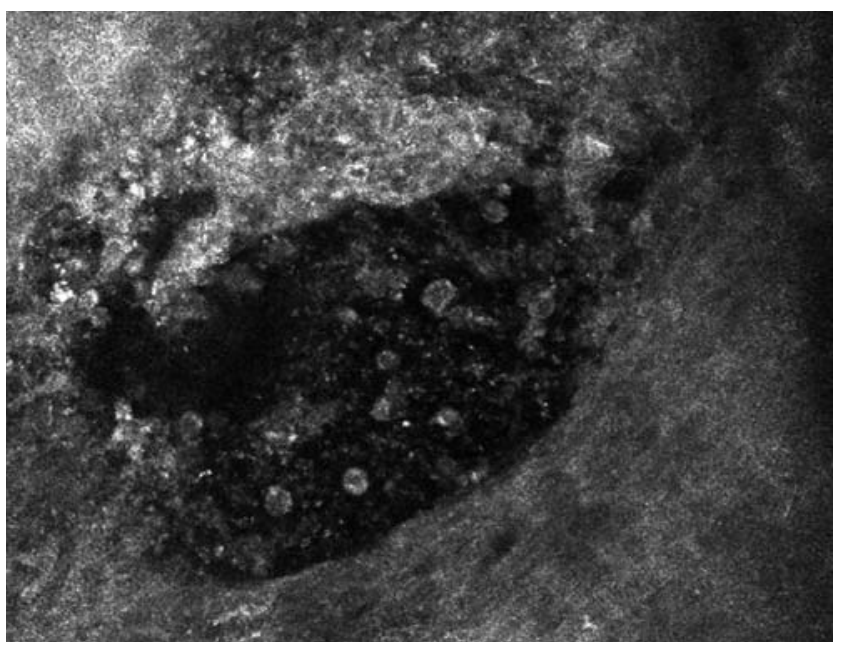

Figura 10: Melanócitos atípicos (células redondas brilhantes com núcleo escuro) isoladas no interior da derme papilar

\section{AGRADECIMENTO}

Agradeço ao Prof. Giovanni Pellacani (Professor do Departamento de Dermatologia da Universidade de Modena e Reggio Emilia) por haver cedido algumas das imagens para a confecção deste artigo e a quem sou eternamente grata pela atenciosa e carinhosa acolhida no seu Serviço em Modena.

\section{REFERÊNCIAS}

1. Leiter U, Garbe C. Epidemiology of melanoma and non-melanoma skin câncer- the role of sun. Adv Exp Med Biol. 2008;624:89-103.

2. Pellacani G, Lo Scocco G, Vicenti $M$, Albertini G, Raccagni AA, Baldassari L, et al. Melanoma epidemic across the millenium:time trends of cutaneous melanoma in Emiliana-Romagna (Italy) from 1997 to 2004. J Eur Acad Dermatol Venereol. 2008;22:213-8.

3. Murchie P, Campbell NC. Pigmented lesions, cutaneous melanoma, and future challenges for primary care. Eur J Gen Pract. 2007;13:151-4.

4. Valentin SM, Sánchez JL, Figueroa LD, Nazario CM. Epidemiology of melanoma in Puerto Rico, 1987-2002. P R Health Sci J. 2007;26:343-8.

5. Bafounta $M$, Beauchet A, Aegerter $P$, Saiag $P$. Is dermoscopy (epiluminescence microscopy) useful for the diagnosis of melanoma? Arch Dermatol. 2001;343-1350.

6. Slominski A, Wortsman J, Nickolff B, McClatchey $\mathrm{K}$, Mihm MC, Ross JS. Molecular pathology of malignant melanoma. Am J Clin Pathol. 1998;110:788-94.

7. Shen SS, Zhang PS, Eton O, Prieto VG. Analysis of protein tyrosine kinase expression in melanocytic lesions by tissue array. J Cutan Pathol. 2003;30:539-47.

8. Soyer PH, Argenziano G, Zalaudek I, Corona R, Sera F, Talamini R, et al. Three-point checklist of dermoscopy. Dermatol. 2004;208:27-31.

9. Garbe G, Eigentler TK. Diagnosis e treatment of cutaneous melanoma:state of the art. Melanoma Res 2006; 17:117-27.
10. Gonzalez S, Gill M, Halpern A. Reflectance confocal microscopy of cutaneous tumors. An atlas with clinical, dermoscopic and histological correlations. U.S.A: Informa HealthCare, 2008. 275p.

11. Brazan AL, Landthaler M, Szeimies M. In vivo confocal scaning laser microscopy in dermatology. Lasers Med Sci. 2007;22:73-82.

12. Rezze G, Sá B, Neves RI. Dermatoscopia: o método de análise de Padrões. An Bras Dermatol. 2006;261-8.

13. Menzies SW, Guteney A, Avramidis M, Batrac A, McCarthy WH. Short-term digital surface microscopic monitoring of atypical or changing melanocytic lesions. Arch Dermatol. 2001;137:1583-9.

14. Kittler $H$, Pehamberger $H$, Wolff $K$, Binder $M$. Diagnostic accuracy of melanoma. Lancet Oncol. 2002;159-65.

15. Pellacani G, Guitera P, Longo C, Avramidis M, Seidenari $\mathrm{S}$, Menzies S. The impacto fo in vivo reflectanc confocal microscopy for the diagnostic accuracy of melanoma and equivocal melanocytic lesions. J Invest Dermatol. 2007; 127:2759-65.

16. Gerger A, Koller S, Weger W, Richtig E, Kerl H, Samonigg H, et al. Sensitivity and specificity of confocal laser-scanning microscopy for in vivo diagnosis of malignant skin tumors. Cancer. 2006;193-200.

17. Langley R, Rajadhyaksha M, Dwyer P, Soer A, Flotte T, Anderson R. Confocal scanning laser microscopy of benign and malignant melanocytic skin lesions in vivo. 
J Am Acad Dermatol. 2001:45:365-76.

18. Gerger A, Hofmann-Wellenhof R, Langsenlehner U, Richtig E, Koller S, Weger W, et al. In vivo confocal laser scanning microscopy of melanocytic skin tumours: diagnostic applicability using unselected tumour images. Br J Dermatol. 2008;158:329-33.

19. Pellacani G, Cesinaro A, Grana C, Seidenari S. In vivo confocal scanning laser microscopy of pigmented spitz nevi: Comparison of in vivo confocal images with dermoscopy and routine histopathology. J Am Acad Dermatol. 2004;51:371-6.

20. Pellacani G, Longo C, Malvehy J, Puig S, Carrera C, Segura $S$, et al. In vivo confocal microscopic and histopathologic correlations of dermoscopic features in 202 melanocytic lesions. Arch Dermatol. 2008;144:1597-608.

21. Segura S, Pellacani G, Puig S, Longo C, Bassoli S, Guiterra P, et al. In vivo microscopic features of nodular melanomas. Arch Dermatol. 2008;144:1311-20.

22. Pellacani $G$, Cesinaro A, Seidenari S. In vivo assessment of melanocytic nests in nevi and melanomas by reflectance confocal microscopy. Mod Pathol. 2005;469-74.

23. Pellacani G, Cesinaro A, Seidenari S. In vivo confocal reflectance microscopy for the characterization of melanocytic nests and correlation with dermoscopy and histology. Br J Dermatol. 2005;152:368-403.

24. Pellacani G, Longo C, Ferrara G, Cesinaro AM, Bassoli S, Guitera P, et al. Spitz nevi: In vivo confocal microscopic features, dermatoscopic aspects, histopathologic correlates, and diagnostic significance. J Am Acad Dermatol. 2009;60:236-47.

25. Scope A, Benvenuto-Andrade C, Agero A, Halpern A, Gonzalez S, Marghoob A. Correlation of dermoscopic structures of melanocytic lesions to reflectance confocal microscopy. Arch Dermatol. 2007;143:176-85.

26. Charles C, Marghoob A, Busam K, Clark-Loeser L, Halpern A. Melanoma or pigmented basal cell carcinoma: a clinical-pathologic correlation with dermoscopy, in vivo confocal scanning laser microscopy, and routine histology. Skin Res Technol. 2002;8:282-7.

27. Pellacani G, Cesinaro A, Longo C, Grana C, Seidenari S. Microscopic in vivo description of cellular architecture of dermoscopic pigment network in nevi and melanomas. Arch Dermatol. 2005;141:147-54.

28. Ahlgrimm-Siess A, Massone C, Koller S, Fink-Puches R, Richtig E, Wolf I, et al. In vivo confocal scanning laser microscopy of common naevi with globular, homogeneous and reticular pattern in dermoscopy. $\mathrm{Br} \mathrm{J}$ Dermatol. 2008;158:1000-07.

29. Minsky M. Microscopy Apparatus. US Patent. $1961 ; 3013467$.

30. Minsky M, Memoir on inventing the confocal scanning microscope. Scanning. 1988;10:123-8.

31. Gonzalez S, Swindells K, Rajadhyaksha M, Torres A.
Changing paradigms in dermatology: Confocal microscopy in clinical and surgical dermatology. Clin Dermatol. 2003;21:359-369.

32. Busan K, Hester K, Charles C, Sachs D, Antonescu C, Gonzalez S, et al. Detection of clinically amelanotic malignant melanoma and assessment of its margins by in vivo confocal scanning laser microscopy. Arch Dermatol. 2001;37:923-929.

33. Tannous Z, Mihm M, Flotte T, Gonzalez S. In vivo examination of lentigo maligna and malignant melanoma in situ, lentigo maligna type by near-infrared reflectance confocal microscopy:Comparison of in vivo confocal images with histologic sections. J Am Acad Dermatol. 2002;46:260-3.

34. Tannous $\mathrm{Z}$, Torres A, Gonzalez S. In vivo real-time confocal reflectance microscopy:A noninvasive guide for Mohs Micrographic Surgery facilitated by aluminum chloride, an excellent contrast enhancer. Dermatol Surg. 2003;29:839-846.

35. Yamashita T, Kuwahara T, Gonzalez S, Takahashi M. Non invasive visualization of melanina and melanocytes by reflectance-mode confocal microscopy. J Invest Dermatol. 2005;124:235-240.

36. Pellacani G, Cesinaro AM, Seidenari S. "ReflectanceMode Confocal Microscopy of Pigmented Skin Lesions Improvement in Melanoma Diagnostic Specificity". J Am Acad Dermatol. 2005;53:979-85.

37. Skin confocal microscopy.org [homepage]. [acesso 10 nov 2008]. Disponível em: http://www.skinconfocalmicroscopy.org/.

38. Busam KJ, Charles C, Lohmann CM, Marghoob A, Goldgeier M, Halpern AC. Detection of intraepidermal malignant melanoma in vivo by confocal scanning laser microscopy. Melanoma Res. 2002; 12:349-55

39. Gerger A, Koller S, Kern T, Massone C, Steiger K, Richtig E, et al. Diagnostic applicability of in vivo confocal laser scanning microscopy in melanocytic skin tumors. J Invest Dermatol. 2005;124:493-8.

40. Pellacani G, Cesinaro AM, Seidenari S. Reflectancemode confocal microscopy for the in vivo characterization of pagetoid melanocytosis in melanoma and nevi. J Invest Dermatol. 2005;125:532-7.

\author{
ENDEREÇO PARA CORRESPONDÊNCIA / MAILING ADDRESS: \\ Cintia Rito \\ Rua Orcadas 447, apto 201, Jardim Carioca, Ilba \\ do Governador, Rio de Janeiro \\ Tel.:/Fax: 21 2463-2938, 7892-5693, 2463-2938 \\ e-mail: cintiarito@botmail.com
}

